\title{
Rat hindlimb joint immobilization with acrylic resin orthoses
}

C.A. da Silva, R.R.J. Guirro,

M.L.O. Polacow,

K.M. Cancelliero and J.L.Q. Durigan
Programa de Pós-Graduação, Departamento de Fisioterapia, Universidade M etodista de Piracicaba, Piracicaba, SP, Brasil

\section{Correspondence \\ R.R.J. Guirro \\ Universidade Metodista de Piracicaba \\ Rodovia do Açúcar, km 156 \\ 13400-911 Piracicaba, SP \\ Brasil \\ E-mail: rjguirro@ unimep.br}

Research supported by FAP/UNIMEP.

Received October 21, 2005

Accepted April 10, 2006

\section{Abstract}

The objective of the present study was to propose an orthosis of light material that would be functional for the animal and that would maintain only the ankle joint immobilized. Male Wistar rats (3 to 4 months old, 250-300 g) were divided into 2 groups $(\mathrm{N}=6)$ : control and immobilized for 7 days. Rats were anesthetized with sodium pentobarbital (40 mg/kg weight) and the left hindlimb was immobilized with the orthoses composed of acrylic resin model, abdominal belt and lateral supports. The following analyses were performed: glycogen content of the soleus, extensor digitorum longus, white gastrocnemius, red gastrocnemius, and tibialis anterior muscles by the phenol sulfuric method, and the weight, fiber area and intramuscular connective tissue of the soleus by the planimetric system. Data were analyzed statistically by the Kolmogorov-Smirnov, Student $t$ and Wilcoxon tests. Immobilization decreased glycogen in all muscles (P $<0.05$; soleus: $31.6 \%$, white gastrocnemius: $56.6 \%$, red gastrocnemius: $39 \%$, extensor digitorum longus: $41.7 \%$, tibialis anterior: $45.2 \%$ ) in addition to reducing soleus weight by $34 \%(\mathrm{P}<0.05)$. Furthermore, immobilization promoted reduction of the fiber area $(43 \%, \mathrm{P}<0.05)$ and increased the connective tissue $(200 \%, \mathrm{P}<0.05)$. The orthosis model was efficient comparing with another alternative immobilization model, like plaster casts, in promoting skeletal muscle alterations, indicating that it could be used as a new model in other studies related to muscle disuse.

\section{Introduction}

Muscular hypotrophy induced by disuse is a condition frequently found in the physiotherapeutic clinic which can occur in association with orthopedic disorders such as ligament ruptures, bone fractures, muscular and medulla lesions, inflammatory processes, degenerative joint and muscular pathologies, as well as in situations in which patients are
Key words

- Immobilization

- Joint

- Orthoses

- Physical therapy
- Hypotrophy

...................... confined to bed for long periods of time for medical or surgical reasons (1).

Joint immobilization induces harmful effects such as muscular fiber hypotrophy, increased connective tissue, loss of muscular extensibility, and limitation of joint movement (2). Besides these alterations, Hirose et al. (3) observed the insulin resistance caused by immobilization compromising the insulin signaling responsible for glycogen synthesis. 
Although immobilization has been extensively studied, more investigation is still required because of the wide variation in responses resulting from joint position, number of joints immobilized, application time, material used, and whether or not there is load on the limb.

A frequently used model is limb suspension, in which there is no weight load, and several studies have associated this model with other techniques. Nemirovskaya and Shenkman (4) associated hindlimb suspension with ankle immobilization in a neutral position and a platform to lean the limb on. In the Tanaka et al. (5) study, suspension was associated with fixing the ankle with steel wire.

The models described in the literature induce a joint position that permits the muscle to remain in a stretched or shortened condition. Some studies have used the plantar flexion position to maintain the soleus muscle in the shortened position, with the extensor digitorum longus and tibialis anterioris muscles thus remaining in a stretched position (6-8). Wagatsuma et al. (9) induced ankle plantar flexion by cast application, maintaining the joint in the maximum position. Sakakima et al. (10) used $70^{\circ}$ positioning and Ahtikoski et al. (6), in addition to studying a group that maintained plantar flexion (150-160 ${ }^{\circ}$, also used a group maintaining ankle dorsiflexion $\left(30-40^{\circ}\right)$. The neutral position of the ankle joint is also used to keep the muscles in a resting condition without muscular tension $(3,11,12)$.

These studies also differed in terms of the type of material type used for joint immobilization. In the Wagatsuma and Yamada (13) study, epoxy resin was used to maintain ankle plantar flexion. In other studies, the neutral position of the ankle differed according to the choice of material, such as hexcelite or fixed needles $(3,11)$ or plaster casts applied for different periods of time $(3,12)$.

In 2002, Coutinho et al. (14) proposed the use of cotton tissue, steel mesh and adhe- sive tape to analyze the soleus, which remained shortened, and the tibialis anterioris, which was stretched. Jarvinen et al. (8) immobilized rat left hindlimbs with casts, maintaining the knee in flexion and the ankle in extension, or vice versa, using the contralateral limb as control.

An aspect observed in the immobilization studies is that more than one joint was immobilized, including the ankle, the knee, the hip, and even the pelvis. Thus, the aim of the present study was to propose an orthosis of light material that would be functional for the animal and that would maintain only one joint immobilized, in this case the ankle kept in a neutral position, leaving the knee and hip joints free, allowing weight load.

\section{Material and Methods}

\section{Orthosis preparation}

Orthosis preparation followed several stages used in dentistry, related to the manufacturing of dental prostheses. Traditionally, acrylic molding models and temporary prostheses are made according to the Phillips methodology (15) that involves the following steps:

1) Molding: the animals were anesthetized with sodium pentobarbital ( $40 \mathrm{mg} / \mathrm{kg}$ weight) and the ankle joint was positioned with a string (Figure 1A and B). For molding, potassium alginate was used (Figure 1C) up to the gelation point, in an amount sufficient to cover and to remove the limb impression. Final gelation and the development of elastic properties were then allowed to occur. 2) Modeling: after gelation, a lateral incision was made and the limb was removed. The parts that were molded on limb removal were brought together and bonded for modeling. Plaster stone was worked with a spatula and poured into the mold under constant agitation to reduce air bubbles (Figure 1D). The final condition was determined to reduce fragility until the alginate solid state. 3) Model detrition: the plaster model (Figure 
1E) was separated from the alginate and its parts were scraped and sanded. 4) Expulsivity: with a lamp and spatula No. 7, wax was placed inside the retention points to create expulsivity areas, but without affecting the anatomical shape of the limb. 5) Acrylic resin application: the plaster model was dipped in an insulator to create an insulator film. After drying, the methyl methacrylate polymer (powder) and the monomer (liquid) were mixed in a glass container to produce the following phases: sandy, sticky, plastic, and rubbery. In the sticky phase, the resin was transferred from the container to the surface of the plaster model and fitted, sculpted and adjusted to the model by hand and with a small Lecron spatula. 6) Acrylization: the acrylic resin was applied to the model, chemically activated, and tested for exothermal reaction. 7) Expulsion: after acrylization, the acrylic resin model (Figure 1F) was polished with stone and polishing eraser and cut laterally with a carbaryl disk. The retention angles were reduced with a number 5 drill and polishing stone.

\section{Immobilization procedure}

Male Wistar rats (3 to 4 months old, 250$300 \mathrm{~g}$ ) were maintained in controlled animal house conditions with free access to food and water and treated in accordance with the recommendations of the Guide on the care and use of laboratory animals (16). The animals were placed in groups of 3 in $40 \times 30$ $\mathrm{cm}$ boxes lined with newspaper in order to prevent sawdust from entering the internal compartment of the orthoses and cause skin lesions.

The animals were divided into two groups $(\mathrm{N}=6)$ : control and immobilized for 7 days. To prepare the orthoses, the rats were anesthetized with sodium pentobarbital $(40 \mathrm{mg} /$ $\mathrm{kg}$ weight) and their left hindlimbs were immobilized with the acrylic resin models (Figure 2) adapted in combination with polyvinyl chloride or vinyl belts $40 \mathrm{~mm}$ in diameter (Figure 1G) covered with latex and at-
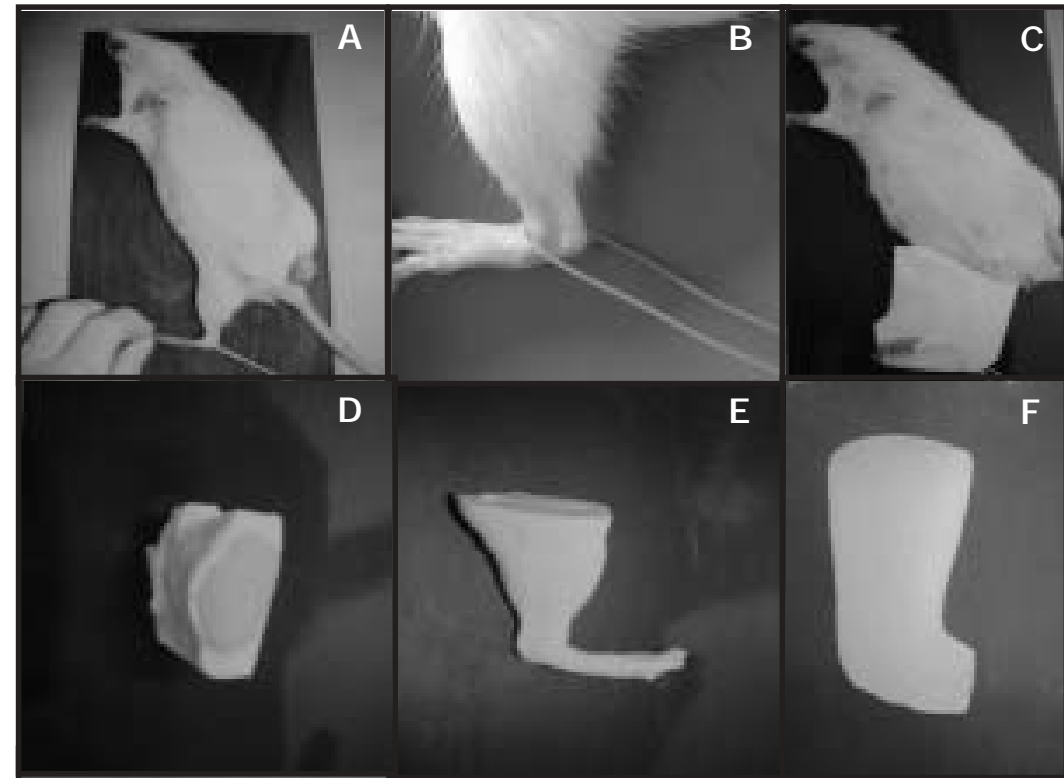

E

$\mathbf{F}$

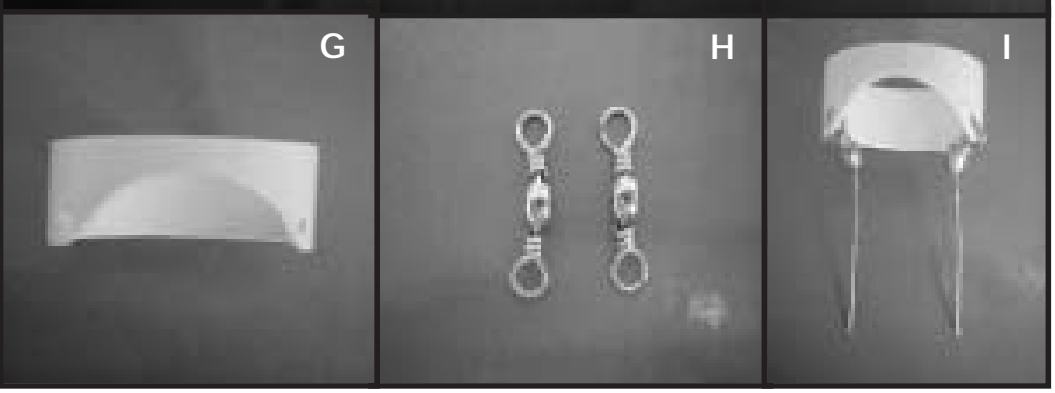

Figure 1. Sequence of the process or orthosis preparation. A and B, positioning of the ankle joint in a neutral position; $C$, limb molding with potassium alginate; $D$, placement of the plaster in the alginate mold; $E$, plaster mold; $F$, acrylic resin model; $G$, abdominal belt; $H$, supports; I, bilateral supports coupled to the abdominal belt.

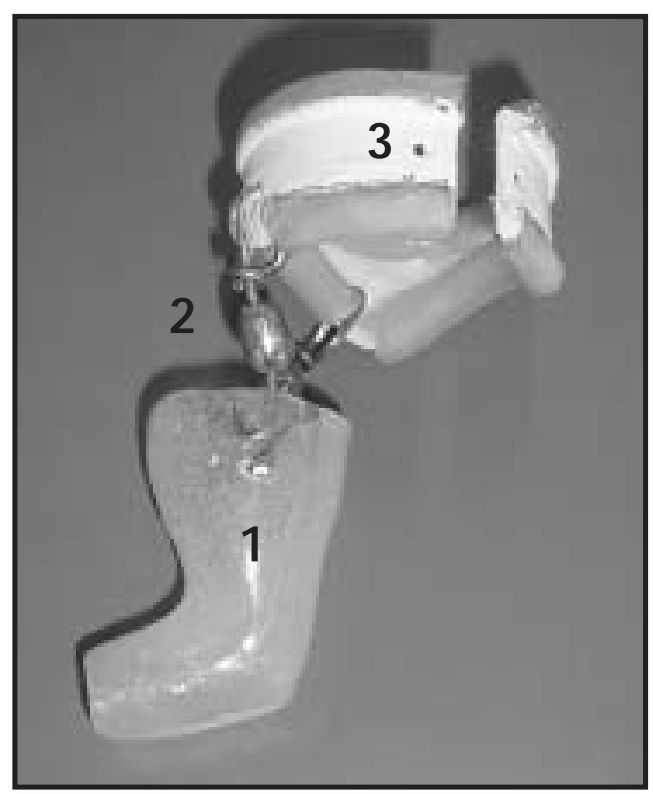

Figure 2. Orthosis in the neutral ankle position consisting of an acrylic resin model (1), lateral rotators (2) and an abdominal belt (3). 
Figure 3. Orthosis adaptation to the animal's left hindlimb, maintaining the ankle joint in a neutral position (A) and placing the weight load on the hindlimb, without interfering with walking (B).

Table 1. Effect of immobilization on muscle glycogen concentration, soleus fiber and connective tissue area of rat submitted to joint immobilization for 7 days.

\begin{tabular}{lcc}
\hline & Control & Immobilized \\
\hline Glycogen (mg/100 mg muscle) & & \\
Soleus & $0.38 \pm 0.06$ & $0.26 \pm 0.03^{*}$ \\
White gastrocnemius & $0.46 \pm 0.06$ & $0.20 \pm 0.05^{*}$ \\
Red gastrocnemius & $0.41 \pm 0.04$ & $0.25 \pm 0.09^{*}$ \\
Extensor digitorum longus & $0.36 \pm 0.08$ & $0.21 \pm 0.06^{*}$ \\
Tibialis anterior & $0.31 \pm 0.09$ & $0.19 \pm 0.07^{*}$ \\
Soleus fiber area $\left(\mu \mathrm{m}^{2}\right)$ & & \\
1st quartile & 2180 & 1197 \\
Median & 2496 & $1423^{*}$ \\
3rd quartile & 2879 & 1659 \\
Soleus connective tissue area (\%) & & \\
1st quartile & 7.1 & 25.0 \\
Median & 8.9 & 30.4 \\
3rd quartile & 10.7 & \\
\hline
\end{tabular}

Data are reported as means \pm SD (glycogen) and medians (fiber and connective tissue area) for 6 animals per group.

*P $<0.05$ compared to control (Kolmogorov-Smirnov, Student t-test, Wilcoxon test). ported as $\mathrm{mg} / 100 \mathrm{mg}$ wet weight.

For morphometric analysis, the ventral segment of the soleus was fixed in buffered $10 \%$ formol solution. The tissue was embedded in paraffin, cut into several non-serial 7 $\mu \mathrm{m}$ thick cross-sections and stained with hematoxylin-eosin.

The image analysis system used consisted of the Image Pro-plus 4.0 software (Media Cybernetics, Silver Spring, MD, USA) and a digital camera (JVC ${ }^{\circledR}$ manufacturer, Lawrenceville, GA, USA) coupled to a microscope (Zeiss, Narberth, PA, USA) and connected to a microcomputer. All the images were captured at 10X magnification.

The areas of 375 soleus muscle fibers from each animal were analyzed as follows: 15 fibers per area in 5 areas per section, and a total of 5 sections per animal. A square reticulum was used for randomly choosing 15 fibers per cuts that coincided with the straight intersections.

A planimetry system was used for the analysis of intramuscular connective tissue density by scoring points by means of a reticulum with $2500-\mu \mathrm{m}^{2}$ squares containing 56 straight line intersections. The coincident points in the endomysium and perimysium in 5 areas per section in 5 sections per animal corresponded to a total of 1400 points per animal.

The relative area of connective tissue (area density) was calculated by dividing the sum of the number of coincident points in the straight line intersections in connective tissue (endomysium and perimysium) by the total number of points.

Although five hindlimb muscles were chosen for metabolic analysis, only the soleus was used for morphometric analysis because, by being monoarticular and by predominantly containing type I fibers, it is the muscle that presents the highest degree of atrophy.

Statistical analysis was initially performed by the Kolmogorov-Smirnov normality test. The muscle weight and glycogen data, which 
presented normal distribution, were analyzed by the Student $t$-test, whereas the data concerning muscle fiber area and connective tissue density, which did not present normal distribution, were analyzed by the nonparametric Wilcoxon test. The level of significance was set at $\mathrm{P}<0.05$ for all analyses.

\section{Results}

The skeletal muscle of rats submitted to hindlimb immobilization for 7 days presented a significant decrease $(\mathrm{P}<0.05)$ in glycogen content indicated by a reduction of $31.6 \%$ in the soleus, $56.6 \%$ in the white gastrocnemius, $39 \%$ in the red gastrocnemius, $41.7 \%$ in the extensor digitorum longus, and $45.2 \%$ in the tibialis anterior muscles, suggesting a functional integration between homeostasis in the fiber contractile process and carbohydrate control (Table 1).

The soleus muscle, which was chosen for weight evaluation, showed a $34 \%$ reduction in weight (control: $123.5 \pm 5.28 \mathrm{mg}$, immobilized: $81.3 \pm 4.63 \mathrm{mg}, \mathrm{P}<0.05$ ), suggesting proteolysis resulting from disuse and osmotic mobilization of active energy reserves.

Immobilization also promoted alterations in the morphology of the soleus muscle, characterized by a $43 \%$ reduction in fiber area $(\mathrm{P}<0.05)$ and by a $200 \%$ increase in connective tissue $(\mathrm{P}<0.05$; Table 1, Figure 4A,B).

The orthosis, which weighed $22.72 \pm$ $2.25 \mathrm{~g}$ (mean $\pm \mathrm{SD}$ ), did not interfere with the animal's ability to walk since its weight was thrown onto the immobilized limb, with a blocked limb movement and anterior and lateral hip movements. It is important to emphasize that the animals showed no change in fluid or solid intake, or any skin lesions in the limb.

\section{Discussion}

The effectiveness of the orthoses in pro- moting metabolic and morphologic alterations in the hindlimb was observed after a period of 7 days. Previous studies had demonstrated that most changes in the skeletal muscle system occur during the first seven days of muscle disuse (18). It has been observed that the greater vulnerability of the slow (type I) compared to the fast muscle fibers (type II) is due to metabolic differences. Immobilization seems to have different effects on protein synthesis by the different types of muscle fibers (7). In has been reported that oxidative enzymes respond with decreased activity during immobilization, suggesting that the muscle fibers with predominantly oxidative metabolism (type I) are more vulnerable to muscle atrophy (19).

In addition to the susceptibility to inherent atrophy of type I fiber metabolism, other factors that determine this condition are the characteristics of postural fibers. Ploug et al. (11) reported that the greater susceptibility of the soleus to atrophy is related to inactivity because it is a postural muscle that has greater basal activity than non-postural muscles. In a recent study, it was observed that immobilization for two weeks, in addition to promoting significant reduction of slow fibers (type I), also promoted a significant increase in fast muscle fibers (type IIc) compared to control (5).

These studies corroborate Lieber's affirmation (20) and support the findings of other studies showing that the muscles considered

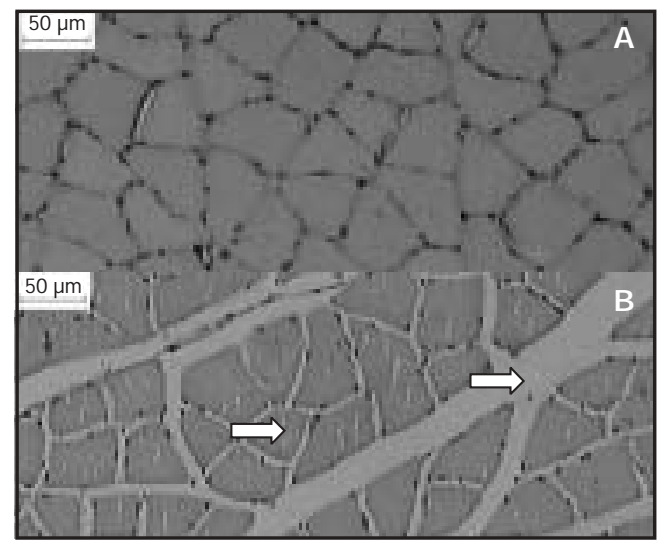

Figure 4. Soleus muscle fibers of the control (A) and immobilized group (B) kept in the neutral ankle position for 7 days. The fibers were stained with hematoxylin and eosin. A reduction of muscle fiber area and an increase of intramuscular connective tissue can be seen in the muscle of the immobilized rat (arrows). 
to be antigravitational, monoarticular and possessing a larger proportion of slow fibers, are the most vulnerable to atrophy induced by muscle disuse.

On this basis, we chose the soleus muscle, predominantly composed of type I fibers, for morphological analysis because of its greater susceptibility to muscle atrophy from disuse. The analysis of glycogen content was also done in this muscle, in addition to the others that compose the triceps sural and anterior compartment of the hindlimb.

The immobilized soleus presented a significant reduction in weight, in glycogen reserves and in fiber area, as well as an increase in connective tissue, showing the interrelation of contractile activity with energy homeostasis and muscle fiber morphology that would appear to indicate a condition of muscle atrophy. Similar results were reported by Fournier et al. (21) who immobilized the soleus and gastrocnemius muscles in shortened, neutral and stretching positions. Their results showed that the muscles immobilized in the shortened and neutral positions lost a significant amount of weight after 28 days. There was a weight reduction of $55 \%$ in the soleus muscle in the neutral position and $77 \%$ in the shortened position; in the gastrocnemius muscle the reduction was of $54 \%$ in the neutral position and of $53 \%$ in the shortened position compared to control.

Generally speaking, immobilization for different periods of times promotes atrophy ranging from 15 to $70 \%$, depending on the animal species and on the fibers evaluated (12). Kannus et al. (22) showed a reduction of $69 \%$ in the fiber area of the soleus immobilized with a plaster cast for 3 weeks.

Regarding the connective tissue of the soleus muscle, the present results are similar to those of other investigations that showed an increase in density in the immobilized muscle condition. Williams et al. (2) observed an increase in the amount of connective tissue in the perimysium of the soleus muscle immobilized in the shortened position for 2 days. They also observed that the collagen fibers of the perimysium presented a more acute angle than observed in normal muscles, with consequent decreased muscular elasticity and increased passive tension.

Other studies have shown morphological alterations caused by immobilization, demonstrating that the increase in connective tissue occurs in the endomysium and perimysium (23). Others also observed that there is increased collagen turnover in the connective tissue during immobilization (22). Indisputably, the amount of intramuscular connective tissue increased dramatically in various disuse models (50-700\%) during immobilization, tenotomy or denervation (23).

Muscle disuse caused by conditions of long periods in bed, orthoses or fixations on limbs and unweighted conditions induce insulin resistance and a catabolic state in the affected skeletal muscles of humans. However, it is still unclear how chronic muscle disuse or immobilization alters insulin signaling (3). Insulin resistance explains the compromised glycogen reserves due to immobilization in all muscles analyzed in the present study.

Hirose et al. (3) studied the signaled insulin pathway in rats that had their left hind limb immobilized by fixation of the knee and ankle to $90^{\circ}$ for 7 days, and verified a reduction in the transduction of the intracellular signal stimulated by insulin, suggesting deficit in insulin receptor activation, and in other molecules including insulin receptor substrate 1 phosphorylation and activation of phosphatidylinositol 3-kinase, indicating that insulin resistance can also be caused by immobilization.

Another important factor is that the orthoses allowed the animal to be functional due to their structure, good adaptation and lightness, with only the ankle joint being immobilized, a situation closer to reality. However, orthoses did not promote skin lesions or edema and the material proved to be 
resistant to the movements of the animal. In addition to promoting skeletal muscle alterations, the type of rat hindlimb immobiliza- tion proposed here, due to it structure, also allowed the animal to be functional, thus deserving further study.

\section{References}

1. Reardon KA, Davis J, Kapsa RM, Choong P, Byrne E. Myostatin, insulin-like growth factor-1, and leukemia inhibitory factor mRNAs are upregulated in chronic human disuse muscle atrophy. Muscle Nerve 2001; 24: 893-899.

2. Williams PE, Catanese T, Lucey EG, Goldspink G. The importance of stretch and contractile activity in the prevention of connective tissue accumulation in muscle. J Anat 1988; 158: 109-114.

3. Hirose M, Kaneki M, Sugita H, Yasuhara S, Martyn J A. Immobilization depresses insulin signaling in skeletal muscle. Am J Physiol Endocrinol Metab 2000; 279: E1235-E1241.

4. Nemirovskaya TL, Shenkman BS. Effect of support stimulation on unloaded soleus in rat. Eur J Appl Physiol 2002; 87: 120-126.

5. Tanaka $T$, Kariya $Y$, Hoshino $Y$. Histochemical study on the changes in muscle fibers in relation to the effects of aging on recovery from muscular atrophy caused by disuse in rats. J Orthop Sci 2004; 9: 7685.

6. Ahtikoski AM, Koskinen SO, Virtanen P, Kovanen V, Takala TE. Regulation of synthesis of fibrillar collagens in rat skeletal muscle during immobilization in shortened and lengthened positions. Acta Physiol Scand 2001; 172: 131-140.

7. Heslinga J W, te Kronnie G, Huijing PA. Growth and immobilization effects on sarcomeres: a comparison between gastrocnemius and soleus muscles of the adult rat. Eur J Appl Physiol Occup Physiol 1995; 70: 49-57.

8. Jarvinen MJ, Einola SA, Virtanen EO. Effect of the position of immobilization upon the tensile properties of the rat gastrocnemius muscle. Arch Phys Med Rehabil 1992; 73: 253-257.

9. Wagatsuma A, Fujimoto K, Yamada S. Effect of treatment with nifedipine, an L-type calcium channel blocker, on muscular atrophy induced by hindlimb immobilization. Scand J Med Sci Sports 2002; 12: $26-30$.

10. Sakakima H, Yoshida $Y$, Sakae K, Morimoto N. Different frequency treadmill running in immobilization-induced muscle atrophy and ankle joint contracture of rats. Scand J Med Sci Sports 2004; 14: 186-192.

11. Ploug T, Ohkuwa T, Handberg A, Vissing J, Galbo H. Effect of immobilization on glucose transport and glucose transporter expression in rat skeletal muscle. Am J Physiol 1995; 268: E980-E986.

12. Qin L, Appell HJ, Chan KM, Maffulli N. Electrical stimulation prevents immobilization atrophy in skeletal muscle of rabbits. Arch Phys Med Rehabil 1997; 78: 512-517.

13. Wagatsuma A, Yamada S. Specific protein alteration in the soleus following immobilization-atrophy. Scand J Med Sci Sports 2000; 10: 205-210.

14. Coutinho EL, Gomes AR, Franca CN, Salvini TF. A new model for the immobilization of the rat hind limb. Braz J Med Biol Res 2002; 35: 1329-1332.

15. Phillips RW. Materiais dentários de skinner. Rio de Janeiro: Guanabara Koogan; 2001.

16. National Research Council. Guide for the care and use of laboratory animals. Washington: National Academy Press; 1996.

17. Lo S, Russell J C, Taylor AW. Determination of glycogen in small tissue samples. J Appl Physiol 1970; 28: 234-236.

18. Okita M, Yoshimura T, Nakano J, Motomura M, Eguchi K. Effects of reduced joint mobility on sarcomere length, collagen fibril arrangement in the endomysium, and hyaluronan in rat soleus muscle. J Muscle Res Cell Motil 2004; 25: 159-166.

19. Appell HJ. Muscular atrophy following immobilisation. A review. Sports Med 1990; 10: 42-58.

20. Lieber RL. Skeletal muscle structure, function, and plasticity, the physiological basis of rehabilitation. Philadelphia: Lippincott; 2002.

21. Fournier M, R oy RR, Perham H, Simard CP, Edgerton VR. Is limb immobilization a model of muscle disuse? Exp Neurol 1983; 80: 147-156.

22. Kannus $P$, J ozsa L, J arvinen $T L$, Kvist $M$, Vieno $T$, J arvinen $T A$, et al. Free mobilization and low- to high-intensity exercise in immobilization-induced muscle atrophy. J Appl Physiol 1998; 84: 14181424.

23. J ozsa L, Kannus P, Thoring J, Reffy A, J arvinen M, Kvist M. The effect of tenotomy and immobilisation on intramuscular connective tissue. A morphometric and microscopic study in rat calf muscles. J Bone J oint Surg Br 1990; 72: 293-297. 